\title{
Editorial: Carbon-Based Bifunctional Oxygen Electrocatalysts
}

\author{
Zexing $\mathrm{Wu}^{1}$, Xiankai $\mathrm{Wan}^{2}$, Wei $\mathrm{Jin}^{3}$ and Gengtao $\mathrm{Fu}^{4 *}$ \\ ${ }^{1}$ State Key Laboratory Base of Eco-Chemical Engineering, College of Chemistry and Molecular Engineering Qingdao \\ University of Science and Technology, Qingdao, China, ${ }^{2}$ Department of Chemistry, Research Center for Materials Science, \\ Graduate School of Science, Nagoya University, Nagoya, Japan, ${ }^{3}$ School of Chemical and Material Engineering, Jiangnan \\ University, Wuxi, China, ${ }^{4}$ School of Chemical and Biomedical Engineering, Nanyang Technological University, Singapore, \\ Singapore
}

Keywords: carbon, metal-air batteries, oxygen reduction reaction, oxygen evolution reaction, bifunctional oxygen electrocatalysts

\section{Editorial on the Research Topic}

\section{Carbon-Based Bifunctional Oxygen Electrocatalysts}

With the ever-increasing environmental pollution and energy crisis, the demand for sustainable and environmentally friendly energy conversion and storage technologies has been on the rise. Recently, metal-air batteries have been extensively investigated because of their high theoretical energy density, low cost, environmental benignity, and satisfactory safety and are, thus, considered as promising energy devices (Wang et al., 2014; Fu et al., 2018). However, the sluggish kinetics of oxygen-related reactions, including the oxygen reduction reaction (ORR) and the oxygen evolution reaction (OER), hinders the large-scale practical applications of metal-air batteries (Pan et al., 2018; Wang et al., 2018) Therefore, it is necessary to exploit efficient electrocatalysts that exhibit high ORR and OER activities.

Until now, Pt- and Ir/Ru-based materials exhibit the best electrocatalytic performances

\section{OPEN ACCESS}

Edited and reviewed by: Valeria Conte,

University of Rome Tor Vergata, Italy

*Correspondence:

Gengtao Fu

gengtaofu@gmail.com

Specialty section:

This article was submitted to

Green and Sustainable Chemistry,

a section of the journal

Frontiers in Chemistry

Received: 01 July 2020

Accepted: 09 July 2020

Published: 23 September 2020

Citation:

Wu Z, Wan X, Jin W and Fu G (2020) Editorial: Carbon-Based Bifunctional

Oxygen Electrocatalysts.

Front. Chem. 8:713.

doi: 10.3389/fchem.2020.00713 for the ORR and OER, respectively. Nevertheless, their scarcity and high cost limit their large-scale employment in metal-air batteries. Therefore, it is important to exploit low-cost and earth-abundant electrocatalysts as alternatives to noble metals for the ORR and OER. Among the studied non-precious electrocatalysts, carbon-based nanomaterials have been widely investigated for oxygen electrode reactions because of their numerous advantages such as wide availability, low cost, remarkable electroconductivity, and environmental benignity. However, the electrocatalytic activities of carbon-based nanomaterials are lower than those of noble metals. Recently, various strategies have been adopted to improve the electrocatalytic performance, such as heteroatom (e.g., N, P, S, B, and F) doping and nanostructure design (including the porous structure and multi-dimensional morphology). Heteroatom doping facilitates electronic redistribution and electroconductivity improvement, which, in turn, enhances the electrocatalytic performance (Chen et al., 2019; Zheng et al., 2019). The presence of a porous structure with multi-dimensional morphology is beneficial for promoting catalytic activity by accelerating the diffusion of the electrolyte and the release of the generated gas (Zhang et al., 2017; Hu et al., 2019). Furthermore, in addition to their application as an electrocatalyst for oxygenrelated reactions, carbon-based nanomaterials are considered as ideal matrices to support metal compounds, which can prevent the aggregation of metal nanoparticles and reduce the electrical resistance. Herein, we collected six valuable contributions focusing on the preparation, structure control, and application of various carbon-based electrocatalysts. The outstanding ORR performance of nitrogen-doped porous carbon, designed and developed by (Liu et al., 2019) via the direct pyrolysis of natural cattail fibers, is discussed. The preparation methods proposed by (Wang M. et al., 2020) for various biomass-derived 
catalysts and their catalytic performance for the ORR in alkaline, neutral, and acidic media are summarized. Furthermore, the carbon-based materials serving as matrices to support $\mathrm{Fe}-\mathrm{Ni}_{2} \mathrm{P}$, (Xiao et al., 2019) $\mathrm{Co}_{3} \mathrm{O}_{4}$, (Cheng et al., 2019) $\mathrm{Fe}_{1-\mathrm{x}} \mathrm{S}$, (Wang H. et al., 2019), and Fe/N (Rauf et al., 2020) are also discussed in this Research Topic; all the prepared electrocatalysts were found to exhibit excellent electrocatalytic activities for oxygen electrode reactions. As guest editors, we would like to thank all the authors for their valuable contributions to this Research Topic and all the reviewers for their important and thoughtful

\section{REFERENCES}

Chen, J., Fan, C., Hu, X., Wang, C., Huang, Z., Fu, G., et al. (2019). Hierarchically porous $\mathrm{Co} / \mathrm{CoxMy}(\mathrm{M}=\mathrm{P}, \mathrm{N})$ as an efficient Mott-Schottky electrocatalyst for oxygen evolution in rechargeable Zn-Air batteries. Small 15:1901518. doi: $10.1002 /$ smll.201901518

Cheng, G., Liu, G., Liu, P., Chen, L., Han, S., Han, J., et al. (2019). Nitrogen-doped ketjenblack carbon supported $\mathrm{Co}_{3} \mathrm{O}_{4}$ nanoparticles as a synergistic electrocatalyst for oxygen reduction reaction. Front. Chem. 7:766. doi: $10.3389 /$ fchem. 2019.00766

Fu, G., Tang, Y., and Lee, J.-M. (2018). Recent advances in carbon-based bifunctional oxygen electrocatalysts for Zn-air batteries. ChemElectroChem 5 , 1424-1434. doi: $10.1002 /$ celc. 201800373

Hu, X., Chen, Y., Zhang, M., Fu, G., Sun, D., Lee, J.-M., et al. (2019). Alveolate porous carbon aerogels supported Co9S8 derived from a novel hybrid hydrogel for bifunctional oxygen electrocatalysis. Carbon 144, 557-566. doi: 10.1016/j.carbon.2018.12.099

Liu, Y., Hu, M., Xu, W., Wu, X., and Jiang, J. (2019). Catalytically active carbon from cattail fibers for electrochemical reduction reaction. Front. Chem. 7:786. doi: 10.3389/fchem.2019.00786

Pan, J., Xu, Y., Yang, H., Dong, Z., Liu, H., and Xia, B. (2018). Advanced architectures and relatives of air electrodes in $\mathrm{Zn}$-Air batteries. Adv. Sci. 5:1700691. doi: 10.1002/advs.201700691

Rauf, M., Wang, J., Iqbal, W., Abbas, M., Khan, S. A., Khan, Q. U., et al. (2020). Novel heteroatom-doped Fe/N/C electrocatalysts with superior activities for oxygen reduction reaction in both acid and alkaline solutions. Front. Chem. 8:78. doi: $10.3389 /$ fchem. 2020.00078

Wang, H., Qiu, X., Wang, W., Jiang, L., and Liu, H. (2019). Iron sulfide nanoparticles embedded into nitrogen and sulfur co-doped carbon sphere as a highly active oxygen reduction electrocatalyst. Front. Chem. 7:855. doi: $10.3389 /$ fchem. 2019.00855

Wang, H.-F., Tang, C., and Zhang, Q. (2018). A review of precious-metalfree bifunctional oxygen electrocatalysts: rational design and applications comments and insights. We hope that this Research Topic will provide useful insights for the development of carbon-based bifunctional catalysts and furnish a background for the research related to advanced carbon chemistry in redox reactions.

\section{AUTHOR CONTRIBUTIONS}

All authors listed have made a substantial, direct and intellectual contribution to the work, and approved it for publication.

in $\mathrm{Zn}$-Air batteries. Adv. Funct. Mater. 28:1803329. doi: 10.1002/adfm.2018 03329

Wang, M., Wang, S., Yang, H., Ku, W., Yang, S., Liu, Z., et al. (2020). Carbon-based electrocatalysts derived from biomass for oxygen reduction reaction: a minireview. Front. Chem. 8:116. doi: 10.3389/fchem.2020. 00116

Wang, Z.-L., Xu, D., Xu, J.-J., and Zhang, X.-B. (2014). Oxygen electrocatalysts in metal-air batteries: from aqueous to nonaqueous electrolytes. Chem. Soc. Rev. 43, 7746-7786. doi: 10.1039/C3CS60248F

Xiao, Y., Deng, S., Li, M., Zhou, Q., Xu, L., Zhang, H., et al. (2019). Immobilization of Fe-doped $\mathrm{Ni}_{2} \mathrm{P}$ particles within biomass agarose-derived porous $\mathrm{N}, \mathrm{P}$-carbon nanosheets for efficient bifunctional oxygen electrocatalysis. Front. Chem. 7:523. doi: 10.3389/fchem.2019.00523

Zhang, W., Xu, X., Zhang, C., Yu, Z., Zhou, Y., Tang, Y., et al. (2017). 3D spaceconfined pyrolysis of double-network aerogels containing In-Fe cyanogel and polyaniline: a new approach to hierarchically porous carbon with exclusive $\mathrm{Fe}-\mathrm{Nx}$ active sites for oxygen reduction catalysis. Small Methods 1:1700167. doi: 10.1002/smtd.201700167

Zheng, X., Wu, J., Cao, X., Abbott, J., Jin, C., Wang, H., et al. (2019). N-, P-, and Sdoped graphene-like carbon catalysts derived from onium salts with enhanced oxygen chemisorption for Zn-air battery cathodes. Appl. Catal. B. Environ. 241, 442-451. doi: 10.1016/j.apcatb.2018.09.054

Conflict of Interest: The authors declare that the research was conducted in the absence of any commercial or financial relationships that could be construed as a potential conflict of interest.

Copyright (c) $2020 \mathrm{Wu}, \mathrm{Wan}$, Jin and Fu. This is an open-access article distributed under the terms of the Creative Commons Attribution License (CC BY). The use, distribution or reproduction in other forums is permitted, provided the original author(s) and the copyright owner(s) are credited and that the original publication in this journal is cited, in accordance with accepted academic practice. No use, distribution or reproduction is permitted which does not comply with these terms. 